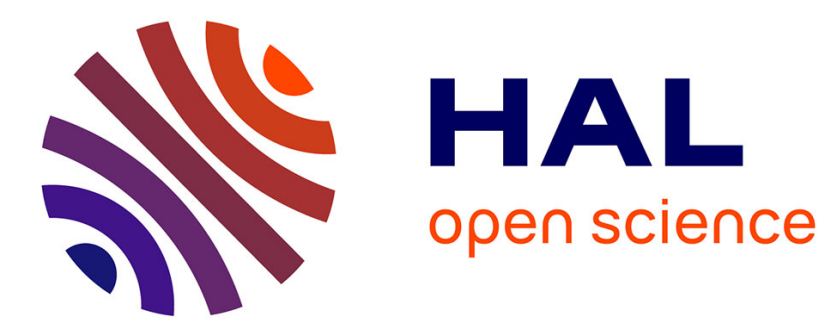

\title{
Generalized reciprocally convex combination lemmas and its application to time-delay systems
}

\author{
Alexandre Seuret, Kun Liu, Frédéric Gouaisbaut
}

\section{To cite this version:}

Alexandre Seuret, Kun Liu, Frédéric Gouaisbaut. Generalized reciprocally convex combination lemmas and its application to time-delay systems. Automatica, 2018, 95, pp.488-493. 10.1016/j.automatica.2018.06.017 . hal-01793309

\section{HAL Id: hal-01793309 \\ https://hal.laas.fr/hal-01793309}

Submitted on 16 May 2018

HAL is a multi-disciplinary open access archive for the deposit and dissemination of scientific research documents, whether they are published or not. The documents may come from teaching and research institutions in France or abroad, or from public or private research centers.
L'archive ouverte pluridisciplinaire HAL, est destinée au dépôt et à la diffusion de documents scientifiques de niveau recherche, publiés ou non, émanant des établissements d'enseignement et de recherche français ou étrangers, des laboratoires publics ou privés. 


\title{
Generalized reciprocally convex combination lemmas and its application to time-delay systems
}

\author{
Alexandre Seuret ${ }^{\mathrm{a}, \mathrm{b}}$, Kun Liu ${ }^{\mathrm{c}}$, Frédéric Gouaisbaut ${ }^{\mathrm{a}, \mathrm{b}}$, \\ ${ }^{\text {a } C N R S, ~ L A A S, ~} 7$ avenue du Colonel Roche, 31077 Toulouse, France. \\ ${ }^{\mathrm{b}}$ Univ de Toulouse, LAAS, F-31400 Toulouse, France. \\ ${ }^{\mathrm{c}}$ School of Automation, Beijing Institute of Technology, 100081 Beijing, China.
}

\begin{abstract}
Various efficient matrix inequalities have recently been proposed to deal with the stability analysis of linear systems with time-varying delays. This paper provides more insights on the relationship between some of them. We present an equivalent formulation of Moon et al.'s inequality, allowing us to discover strong links not only with the most recent and efficient matrix inequalities such as the reciprocally convex combination lemma and also its relaxed version but also with some previous inequalities such as the approximation inequality introduced in [14] or free-matrix-based inequality. More especially, it is proved that these existing inequalities can be captured as particular cases of Moon et al.'s inequality. Examples show the best tradeoff between the reduction of conservatism and the numerical complexity.
\end{abstract}

Key words: Time-delay systems, matrix inequalities, generalized reciprocally convex lemmas.

\section{Introduction}

Providing less conservative and computationally efficient stability conditions for linear systems subject to timevarying delays has attracted considerable attention over the past decades. To deal with integral quadratic terms that arise from the derivative of Lyapunov-Krasovskii functional, two main technical steps, namely the derivation of efficient integral and matrix inequalities are usually adopted. Several attempts have been done concerning integral inequalities such as Jensen [1], Wirtingerbased [10], auxiliary-based [3, 9], Bessel inequalities [11] or polynomials-based inequality [4]. Although these inequalities have shown a great interest for constant delay systems, their application to time/fast-varying delays re-

\footnotetext{
^ This work was partially supported by the ANR project SCIDiS contract number 15-CE23-0014, the National Natural Science Foundation of China (grant no. 61503026, 61720106010), the Beijing Natural Science Foundation (grant no. 4182057), and the Foundation of Beijing Institute of Technology (grant no. 3060011181811). This paper was not presented at any conference. Correspondence of this paper is addressed to K. Liu.

Email addresses: aseuret@laas.fr (Alexandre Seuret), kunliubit@bit.edu.cn (Kun Liu), fgouais@laas.fr (Frédéric Gouaisbaut).
}

veals additional difficulties related to the non-convexity of the resulting terms. Therefore, some matrix inequalities are employed to address this problem and to derive convex conditions. A huge number of papers have studied the ways to combine efficient integral and matrix inequalities. The reader may look for instance to $[5,17]$. Hence, a first method corresponds to the employment of Young's or Moon et al.'s inequalities [7], after the application of an integral inequality. It is also noted that the recent free-matrix-based inequality [16] can be interpreted as the merge of the Wirtinger-based inequality and Moon et al.'s inequality. Furthermore, the reciprocally convex lemma was proposed in [8]. The novelty of this method consists in merging the non-convex terms into a single expression to derive an accurate convex inequality. It was notably shown in [5] that the reciprocally convex combination lemma [8] leads to the same conservatism as the Moon et al.'s inequality when considering Jensenbased stability criteria, but with a lower computational burden. More recently, a relaxed reciprocally convex combination lemma was developed in [19] without requiring any extra decision variable. This inequality was recently extended by the same authors in [18].

The present paper aims at providing more insights on the relationship between some of these bounding methods. We present an equivalent formulation of Moon et 
al.'s inequality, allowing us to discover strong links not only with the most recent and efficient matrix inequalities such as the reciprocally convex combination lemma [8] or its relaxed version [19] but also with some previous inequalities such as $[14,16]$. More especially, we prove that these existing inequalities can be captured as particular cases of Moon et al.'s inequality. Examples show the best tradeoff between the reduction of conservatism and the numerical complexity.

Notations: Throughout the paper, $\mathbb{R}^{n}$ denotes the $n$ dimensional Euclidean space. The notations $\mathbb{R}^{n \times m}$ and $\mathbb{S}^{n}$ are the set of $n \times m$ real matrices and of $n \times n$ real symmetric matrices, respectively. The notation $P \in \mathbb{S}_{+}^{n}$, means that $P \in \mathbb{S}^{n}$ and $P \succ 0$, which means that $P$ is symmetric positive definite. The symmetric elements of a symmetric matrix will be denoted by $*$. For any matrices $A, B$ of appropriate dimension, the matrix $\operatorname{diag}(A, B)$ stands for $\left[\begin{array}{ll}A & 0 \\ 0 & B\end{array}\right]$. The matrices $I_{n}$ and $0_{n, m}$ represent the identity and null matrices of appropriate dimension and, when no confusion is possible, the subscript will be omitted. Moreover, for any square matrix $A$, we define $\operatorname{He}(A)=A+A^{\top}$. For any $h>0$ and any function $x:[-h,+\infty) \rightarrow \mathbb{R}^{n}$, the notation $x_{t}(\theta)$ stands for $x(t+\theta)$, for all $t \geq 0$ and all $\theta \in[-h, 0]$.

\section{Matrix inequalities for systems with time- varying delays}

When considering stability of systems with time-varying delays, the problem often relies on finding a lower bound of a reciprocally convex quadratic term $\Theta$ given by

$$
\Theta(\alpha)=\Gamma^{\top}\left[\begin{array}{cc}
\frac{1}{\alpha} R & 0 \\
0 & \frac{1}{1-\alpha} R
\end{array}\right] \Gamma, \quad \forall \alpha \in(0,1),
$$

where, for given integers $n$ and $m$ such that $2 n \leq m, R$ is in $\mathbb{S}_{+}^{n}, \Gamma$ in $\mathbb{R}^{2 n \times m}$, such that $\operatorname{rank}(\Gamma)=2 n$. There are two main methods to find lower bounds. The first one is based on the Moon et al.'s inequality (see, e.g., the survey paper [15]). The second method is the so-called reciprocally convex combination lemma developed in [8]. The conservatism induced by these two inequalities are independent. While, in some cases, such as stability conditions resulting from the application of the Jensen inequality, these two methods lead to equivalent results on examples [5]. In general, reciprocally convex combination lemma is more conservative than Moon et al.'s inequality (see e.g., $[5,16]$ ).

The objective of this paper is to provide more insights on the relationship between these two classes of bounding methods. In particular, we show that the Moon et al.'s inequality encompasses the reciprocally convex lemmas as particular cases. Moreover, following this idea, we propose a generalization of reciprocally convex lemmas, which again represents a particular case of the Moon et al.'s inequality. This generalization allows providing less restrictive results than the recent extension of the reciprocally convex lemmas $[18,19,20]$.

\section{Modified Moon et al.'s inequality}

\subsection{Main result}

The main result of the paper is stated below. It corresponds to a method to obtain a lower bound of the matrix $\Theta$ defined above, based on Moon et al.'s inequality, which is recalled below.

Lemma 1 [15] For any $x, y \in \mathbb{R}^{n}$, any scalar $\epsilon>0$, any matrix $R$ in $\mathbb{S}_{+}^{n}$, the following inequality holds

$$
2 x^{T} y \leq \epsilon^{-1} x^{T} R x+\epsilon y^{T} R^{-1} y .
$$

The relationship between Moon et al.'s inequality and the reciprocally convex combination lemma has already studied in [5]. The next lemma will extend this work and formulate a generalization of the reciprocally convex combination lemma. The main result of this paper is stated as follows:

Lemma 2 Consider a parameter dependent matrix $\Phi(\alpha)$ in $\mathbb{S}^{m}$, such that the convex inequality

$$
\Phi(\alpha) \preceq(1-\alpha) \Phi(0)+\alpha \Phi(1)
$$

holds for all $\alpha$ in $[0,1]$. If there exist a matrix $R$ in $\mathbb{S}_{+}^{n}$ and two matrices $N_{1}, N_{2}$ in $\mathbb{R}^{m \times n}$ such that the inequality

$$
\Psi(\alpha)=\left[\begin{array}{cc}
\Phi(\alpha)-\Gamma^{\top} \mathcal{R}^{0}(\alpha) \Gamma-\operatorname{He}\left(\Gamma^{\top}\left[\begin{array}{c}
(1-\alpha) N_{1}^{\top} \\
\alpha N_{2}^{\top}
\end{array}\right]\right) * \\
\alpha N_{1}^{\top}+(1-\alpha) N_{2}^{\top}
\end{array}\right] \prec 0
$$

holds for $\alpha=\{0,1\}$, where

$$
\mathcal{R}^{0}(\alpha)=\left[\begin{array}{cc}
(2-\alpha) R & 0 \\
0 & (1+\alpha) R
\end{array}\right]
$$

then, the following inequality holds

$$
\Phi(\alpha)-\Theta(\alpha) \prec 0, \quad \forall \alpha \in(0,1) .
$$

Proof : Let us introduce the following positive quadratic term

$$
\Pi^{\top}(\alpha)\left[\begin{array}{cc}
\frac{1}{\alpha} R^{-1} & 0 \\
0 & \frac{1}{1-\alpha} R^{-1}
\end{array}\right] \Pi(\alpha) \succeq 0,
$$


defined for any $\alpha$ in $(0,1)$, where

$$
\Pi(\alpha)=\left[\begin{array}{cc}
R & 0 \\
0 & R
\end{array}\right] \Gamma-\left[\begin{array}{cc}
\alpha R & 0 \\
0 & (1-\alpha) R
\end{array}\right] \Gamma-\left[\begin{array}{c}
\alpha N_{1}^{\top} \\
(1-\alpha) N_{2}^{\top}
\end{array}\right] .
$$

This inequality indeed holds for any $\alpha$ in $(0,1)$ since the matrix $R$ is assumed to be positive definite and $\alpha$ is positive. Expanding this positive quadratic term leads to

$$
\begin{aligned}
-\Theta(\alpha) \preceq & -\Gamma^{\top} \mathcal{R}^{0}(\alpha) \Gamma-\operatorname{He}\left(\Gamma^{\top}\left[\begin{array}{c}
(1-\alpha) N_{1}^{\top} \\
\alpha N_{2}^{\top}
\end{array}\right]\right) \\
& +\alpha N_{1} R^{-1} N_{1}^{\top}+(1-\alpha) N_{2} R^{-1} N_{2}^{\top}
\end{aligned}
$$

holds for all $\alpha$ in $(0,1)$, where $\Theta(\alpha)$ is defined in (1). Reinjecting the previous expression of $\Theta(\alpha)$ into the left side of (5), we obtain that, for all $\alpha \in(0,1)$,

$$
\begin{gathered}
\Phi(\alpha)-\Theta(\alpha) \preceq \Phi(\alpha)-\Gamma^{\top} \mathcal{R}^{0}(\alpha) \Gamma-\operatorname{He}\left(\Gamma^{\top}\left[\begin{array}{c}
(1-\alpha) N_{1}^{\top} \\
\alpha N_{2}^{\top}
\end{array}\right]\right) \\
+\alpha N_{1} R^{-1} N_{1}^{\top}+(1-\alpha) N_{2} R^{-1} N_{2}^{\top} .
\end{gathered}
$$

Since the right-hand-side of the previous inequality is convex with respect to $\alpha$ and is also well defined for $\alpha=\{0,1\}$, the negative definiteness of $\Phi(\alpha)-\Theta(\alpha)$ is guaranteed if, after application of the Schur complement, $\Psi(0) \prec 0$ and $\Psi(1) \prec 0$. Therefore, if the condition (3) is verified for $\alpha=\{0,1\}$, then the inequality $\Phi(\alpha)-\Theta(\alpha) \prec 0$ holds, for all $\alpha \in(0,1)$.

Remark 1 In some cases, instead of considering the matrix $\Theta(\alpha)$, it might be relevant to consider a matrix $\tilde{\Theta}(\alpha)$ given by

$$
\tilde{\Theta}(\alpha)=\tilde{\Gamma}^{\top}\left[\begin{array}{cc}
\frac{1}{\alpha} \tilde{R}_{1} & 0 \\
0 & \frac{1}{1-\alpha} \tilde{R}_{2}
\end{array}\right] \tilde{\Gamma}, \quad \forall \alpha \in(0,1),
$$

where, for given integers $n_{1}, n_{2}$ and $m$ such that $n_{1}+n_{2} \leq$ $m, \tilde{R}_{1}$ is in $\mathbb{S}_{+}^{n_{1}}, \tilde{R}_{2}$ in $\mathbb{S}_{+}^{n_{2}}, \Gamma$ in $\mathbb{R}^{\left(n_{1}+n_{2}\right) \times m}$, such that $\operatorname{rank}(\tilde{\Gamma})=n_{1}+n_{2}$. It is noted that the matrix $\tilde{\Theta}(\alpha)$ is defined with two matrices, not necessarily of equal dimension. Lemma 2 can be easily extended to this case without any difficulties, and therefore, will not be presented.

\subsection{Comments and interpretations}

The previous Lemma finally demonstrates an equivalence between the inequality presented in Lemma 2 and the usual Moon et al.'s inequality. Indeed taking $N_{1}^{\top}=$ $\tilde{N}_{1}^{\top}-\left[\begin{array}{ll}R & 0\end{array}\right] \Gamma$ and $N_{2}^{\top}=\tilde{N}_{2}^{\top}-\left[\begin{array}{ll}0 & R\end{array}\right] \Gamma$ in (3) allows recovering the usual formulation of Moon et al.'s inequality for systems with time-varying delays. However, this new formulation proposed in Lemma 2 allows us to discover strong links with the most recent and efficient matrix inequalities such as the reciprocally convex combination lemma from [8] or its relaxed version proposed in [19] and also some previous inequalities such as [14], [16]. More especially, we will prove that these existing inequalities can be captured as particular cases of Lemma 2 and, consequently, as particular cases of Moon et al.'s inequality.

In Lemma 2, the number of additional decision variables due to the introduction of $N_{1}$ and $N_{2}$, is $m \times 2 n$. For large scale systems, this number increases in a polynomial manner with respect to $n$ and $m$. Therefore, in order to reduce the complexity of Lemma 2 , it is possible to restrict the number of decision variables by imposing some particular structure to the matrices $N_{1}$ and $N_{2}$. Of course, a restriction on the structure of these two matrices necessarily introduces conservatism. Then a natural question becomes how to find the best tradeoff between the reduction of the complexity (i.e., the number of decision variables) and the reduction of the conservatism (i.e., how far are the numerical results obtained using structured matrices $N_{1}, N_{2}$ from the unstructured case).

Consider, a first example, $N_{1}=N_{2}=0$, which is indeed a conservative case. In this case, inequality (5) is guaranteed for all $\alpha$ in $(0,1)$ if $\Phi(\alpha)-\Gamma^{\top} \mathcal{R}^{0}(\alpha) \Gamma \prec 0$, for $\alpha=\{0,1\}$ holds. This result does not represent a new contribution since it reflects the inequality provided in [14]. Indeed in this paper, the authors use the convexity properties of the inverse function to obtain

$$
\frac{1}{\alpha}=\frac{1}{1-(1-\alpha)} \geq 2-\alpha, \quad \frac{1}{1-\alpha} \geq 1+\alpha .
$$

It is, however, interesting to note that this inequality is already somehow captured in Lemma 2. In the sequel, we propose a corollary of Lemma 2 resulting from two particular selections of matrices $N_{1}, N_{2}$.

\subsection{Generalized reciprocally convex lemmas}

The following corollary aims at presenting particular cases of Lemma 2, which can be seen as extended version of the results of $[18,19,20]$ and also [16].

Corollary 1 Consider a parameter dependent matrix $\Phi(\alpha)$ in $\mathbb{S}^{m}$, such that the convexity inequality (2) is verified. Then inequality (5) is satisfied for all $\alpha$ in $(0,1)$ if one of the following conditions holds

(i) there exist a matrix $R \in \mathbb{S}_{+}^{n}$, two symmetric matrices $Y_{1}, Y_{2} \in \mathbb{S}^{n}$, two matrices $X_{1}, X_{2} \in \mathbb{R}^{n \times n}$ such that the inequality

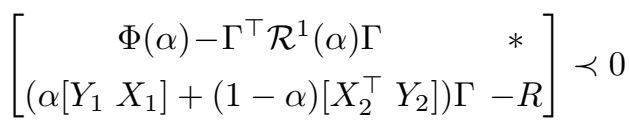


holds for $\alpha=\{0,1\}$, where

$$
\mathcal{R}^{1}(\alpha)=\left[\begin{array}{cc}
(2-\alpha) R+2(1-\alpha) Y_{1} & * \\
(1-\alpha) X_{1}^{\top}+\alpha X_{2}^{\top} & (1+\alpha) R+2 \alpha Y_{2}
\end{array}\right]
$$

(ii) there exist a matrix $R \in \mathbb{S}_{+}^{n}$, two symmetric matrices $Y_{1}, Y_{2} \in \mathbb{S}^{n}$, two matrices $X_{1}, X_{2} \in \mathbb{R}^{n \times n}$, and a matrix $Z \in \mathbb{R}^{n \times n_{0}}$ such that

$$
\begin{aligned}
& \Sigma(\alpha)=
\end{aligned}
$$

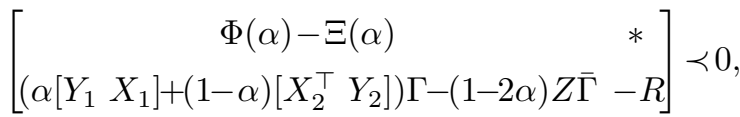

holds for $\alpha=\{0,1\}$, where

$$
\Xi(\alpha)=\left[\begin{array}{l}
\Gamma \\
\bar{\Gamma}
\end{array}\right]^{\top}\left[\begin{array}{cr}
\mathcal{R}^{1}(\alpha) & * \\
{\left[(1-\alpha) Z^{\top}-\alpha Z^{\top}\right]} & 0
\end{array}\right]\left[\begin{array}{l}
\Gamma \\
\bar{\Gamma}
\end{array}\right],
$$

and where $\bar{\Gamma}$ is a matrix in $\mathbb{R}^{n_{0} \times m}, \mathcal{R}^{1}(\alpha)$ is defined in (7).

(iii) there exist a matrix $R \in \mathbb{S}_{+}^{n}$, two symmetric matrices $Y_{1}, Y_{2} \in \mathbb{S}^{n}$, two matrices $X_{1}, X_{2} \in \mathbb{R}^{n \times n}$, a matrix $Z \in \mathbb{R}^{n \times n_{0}}$ and two matrices $W_{1}, W_{2} \in$ $\mathbb{S}^{2 n+n_{0}}$ such that the inequalities

$$
\Psi_{W}(0) \prec 0, \Psi_{W}(1) \prec 0, \Theta_{1} \succeq 0, \Theta_{2} \succeq 0
$$

hold, where

$$
\begin{aligned}
& \Psi_{W}(\alpha)=\Phi(\alpha)-\Xi(\alpha)+\left[\begin{array}{c}
\Gamma \\
\bar{\Gamma}
\end{array}\right]^{\top}\left(\alpha W_{1}+(1-\alpha) W_{2}\right)\left[\begin{array}{l}
\Gamma \\
\bar{\Gamma}
\end{array}\right],
\end{aligned}
$$

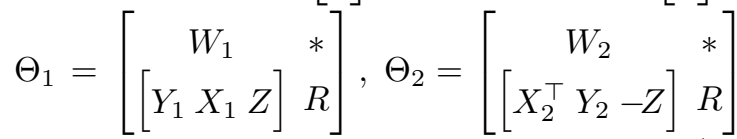

with $\Xi(\alpha)$ defined in (9).

Proof: The proof consists in proving each item separately.

Proof of (i): The proof simply consists in restricting matrices $N_{1}$ and $N_{2}$ to $N_{1}^{\top}=\left[\begin{array}{ll}Y_{1} & X_{1}\end{array}\right] \Gamma, N_{2}^{\top}=\left[\begin{array}{ll}X_{2}^{\top} & Y_{2}\end{array}\right] \Gamma$ in (3).

Proof of (ii): The proof simply consists in taking $N_{1}^{\top}=$

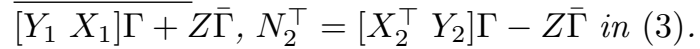

Proof of (iii): Note that conditions $\Theta_{1} \succeq 0$ and $\Theta_{2} \succeq 0$ are equivalent to $W_{1}-\left[\begin{array}{lll}Y_{1} & X_{1} Z\end{array}\right]^{\top} R^{-1}\left[Y_{1} X_{1} Z\right] \triangleq \hat{\Theta}_{1} \succeq$ 0 and $W_{2}-\left[X_{2}^{\top} Y_{2}-Z\right]^{\top} R^{-1}\left[X_{2}^{\top} Y_{2}-Z\right] \triangleq \hat{\Theta}_{2} \succeq 0$, respectively. The latter two inequalities imply that $\alpha \hat{\Theta}_{1}+$ $(1-\alpha) \hat{\Theta}_{2} \succeq 0$ for any $\alpha \in[0,1]$. Moreover, $\Psi_{W}(0) \prec 0$ and $\Psi_{W}(1) \prec 0$ imply $\Psi_{W}(\alpha) \prec 0$ for any $\alpha \in[0,1]$. Then, it follows that

$$
\Psi_{W}(\alpha)-\left[\begin{array}{l}
\Gamma \\
\bar{\Gamma}
\end{array}\right]^{\top}\left(\alpha \hat{\Theta}_{1}+(1-\alpha) \hat{\Theta}_{2}\right)\left[\begin{array}{l}
\Gamma \\
\bar{\Gamma}
\end{array}\right] \prec 0,
$$

which implies

$$
\begin{aligned}
& \hat{\Phi}(\alpha) \triangleq \Phi(\alpha)-\Xi(\alpha) \\
& \left.+\alpha\left[\begin{array}{l}
\Gamma \\
\bar{\Gamma}
\end{array}\right] \quad\left(\begin{array}{lll}
Y_{1} & X_{1} & Z
\end{array}\right]^{\top} R^{-1}\left[\begin{array}{lll}
Y_{1} & X_{1} & Z
\end{array}\right]\right)\left[\begin{array}{c}
\Gamma \\
\bar{\Gamma}
\end{array}\right] \\
& \left.+(1-\alpha)\left[\begin{array}{l}
\Gamma \\
\bar{\Gamma}
\end{array}\right]^{\top}\left(\begin{array}{lll}
{\left[X_{2}^{\top}\right.} & Y_{2} & -Z
\end{array}\right]^{\top} R^{-1}\left[\begin{array}{lll}
X_{2}^{\top} & Y_{2} & -Z
\end{array}\right]\right)\left[\begin{array}{l}
\Gamma \\
\bar{\Gamma}
\end{array}\right] \prec 0 .
\end{aligned}
$$

Since $\hat{\Phi}(\alpha)$ is convex with respect to $\alpha$ and is also well defined for $\alpha=\{0,1\}$, the negative definiteness of $\Sigma(\alpha)$ defined in (8) is guaranteed due to, after application of the Schur complement, $\hat{\Phi}(0) \prec 0$ and $\hat{\Phi}(1) \prec 0$. Hence condition (ii) of Corollary 1 allows us to conclude the proof of (iii).

Remark 2 It is worth mentioning that condition (i) of Corollary 1 extends the relaxed reciprocally convex combination lemma developed in [19] since this lemma can be obtained by selecting, in condition (i), $Y_{1}=Y_{2}=0$ and $X_{1}=X_{2}$. Moreover, it was demonstrated in [19] that the inequality therein is less restrictive than the original reciprocally convex combination lemma developed in [8]. Indeed, condition (i) of Corollary 1 can be interpreted as the following reciprocally convex matrix inequality

$$
\left[\begin{array}{cc}
\frac{1}{\alpha} R & 0 \\
0 & \frac{1}{1-\alpha} R
\end{array}\right] \succeq \mathcal{R}^{1}(\alpha)
$$

Another relaxed version was recently presented in [18, 20] which corresponds to the case where only $Y_{1}=Y_{2}=0$ and where $X_{1}$ and $X_{2}$ are not equal anymore. Note that replacing, in $\mathcal{R}^{1}(\alpha)$ given by ( 7$), R+2 Y_{i}$ by $Y_{i}, i=\{1,2\}$, we obtain that the matrix $\mathcal{R}^{1}(\alpha)$ has the same form as the delay-dependent reciprocally convex combination lemma in [12]. However, there are no additional constraints required on the matrices $Y_{i}, X_{i}, i=\{1,2\}$, in condition (i), unlike in [12], which demonstrates that condition (i) of Corollary 1 is more general.

Remark 3 In conditions (i-ii-iii) of Corollary 1, the matrices $N_{1}$ and $N_{2}$ have been selected with a particular structure that is characterized by the matrix $\Gamma$. This corresponds to a very restrictive selection since $\operatorname{rank}(\Gamma)=$ $2 n \leq m$. Therefore, it is possible to extend condition (i) 
by introducing another matrix $\bar{\Gamma}$ in $\mathbb{R}^{n_{0} \times m}$ such that $\operatorname{rank}\left(\left[\Gamma^{\top} \bar{\Gamma}^{\top}\right]\right)$ is an integer in the interval $[2 n+1, m]$. This leads to condition (ii) of Corollary 1. Moreover, condition (iii) of Corollary 1 is a more general formulation of condition (ii) since condition (iii) is formulated with free-weighted matrices $W_{i}, i=\{1,2\}$.

Furthermore, we also state the following proposition.

Proposition 1 Conditions (ii) and (iii) of Corollary 1 are equivalent.

Proof : Indeed the inequality (10) implies that $\Sigma(\alpha) \prec$ 0 for $\alpha=\{0,1\}$, as demonstrated in the proof of (iii) of Corollary 1. The reverse implication is obtained by noting that, if inequality $\Sigma(\alpha) \prec 0$ holds for $\alpha=\{0,1\}$, there exists a sufficiently small $\epsilon>0$ such that

$$
\Phi(\alpha)-\Xi(\alpha)+\left[\begin{array}{c}
\Gamma \\
\bar{\Gamma}
\end{array}\right]^{\top}\left(\epsilon I+Y^{\top}(\alpha) R^{-1} Y(\alpha)\right)\left[\begin{array}{c}
\Gamma \\
\bar{\Gamma}
\end{array}\right] \prec 0
$$

where $Y(\alpha)=\alpha\left[\begin{array}{lll}Y_{1} & X_{1} & Z\end{array}\right]+\left(\begin{array}{lll}1-\alpha & -\alpha\end{array} X_{2}^{\top} Y_{2}-Z\right]$. Then it is easy to verify that the matrices $W_{1}=$ $\epsilon I+Y^{\top}(1) R^{-1} Y(1)$ and $W_{2}=\epsilon I+Y^{\top}(0) R^{-1} Y(0)$ allow to ensure inequalities (10).

Remark 4 Recently, the free-matrix-based integral inequality that was developed in [16] yields apparently less conservative stability criteria for systems with time-varying delays at least on examples. This freematrix-based inequality leads to conditions presented in Corollary 1 (iii), where matrices $W_{1}$ and $W_{2}$ have been introduced in the design of the integral inequality. In light of Proposition 1, these additional matrices $W_{1}$ and $W_{2}$ are slack variables and can be removed without affecting the numerical results. It therefore only increases the numerical complexity of the conditions. More importantly, it can be seen as a direct application of Moon et al.'s inequality. Similarly, the improved version of [16] presented in [4] is demonstrated to be equivalent to the use of the Bessel-Legendre inequality [11] together with Lemma 2.

\section{Stability analysis of time-varying delay sys- tems}

Consider a linear system with time-varying delays:

$$
\begin{cases}\dot{x}(t)=A x(t)+A_{d} x(t-h(t)), & \forall t \geq 0, \\ x(t)=\phi(t), & \forall t \in\left[-h_{2}, 0\right],\end{cases}
$$

where $x(t) \in \mathbb{R}^{n}$ is the state vector, $\phi$ is the initial condition and $A, A_{d} \in \mathbb{R}^{n \times n}$ are constant matrices. The time-varying delay $h(t)$ is continuous and satisfies

$$
0 \leq h_{1} \leq h(t) \leq h_{2}, \quad h_{12} \triangleq h_{2}-h_{1} .
$$

When possible, the time argument of the delay function $h$ will be omitted. Based on Wirtinger-based inequality proposed in [10] and matrix inequalities analyzed in Corollary 1, the following stability theorem is provided.

Theorem 1 Consider matrices $P$ in $\mathbb{S}_{+}^{3 n}, S_{1}, S_{2}, R_{1}, R_{2}$ in $\mathbb{S}_{+}^{n}$, which define the delay-dependent matrix $\Phi_{0}(\alpha)$ given by

$$
\begin{aligned}
\Phi_{0}(\alpha)= & \operatorname{He}\left(G_{1}^{\top}(\alpha) P G_{0}\right)+\hat{\mathcal{S}}-G_{2}^{\top} \mathcal{R}_{1} G_{2} \\
& +g_{0}^{\top}\left(h_{1}^{2} R_{1}+h_{12}^{2} R_{2}\right) g_{0}, \\
\hat{\mathcal{S}}= & \operatorname{diag}\left(S_{1},-S_{1}+S_{2}, 0_{n},-S_{2}, 0_{3 n}\right), \\
\mathcal{R}_{i}= & \operatorname{diag}\left(R_{i}, 3 R_{i}\right), \quad \forall i=1,2,
\end{aligned}
$$

with matrices $g_{0}, G_{0}, G_{1}, G_{2}$ and the matrix $\Gamma$ given in (16). Then, system (14) is asymptotically stable for all time-varying delays $h$ satisfying (15) if one of the following statement holds for $\alpha=\{0,1\}$ with $R=\mathcal{R}_{2}$ and $\Phi(\alpha)=\Phi_{0}(\alpha)$ :

(i) [19] there exists a matrix $X=X_{1}=X_{2} \in \mathbb{R}^{2 n \times 2 n}$ such that (6) holds with $Y_{1}=Y_{2}=0$.

(ii) $\left[18\right.$, 20] there exist matrices $X_{1}, X_{2} \in \mathbb{R}^{2 n \times 2 n}$ such that (6) holds with $Y_{1}=Y_{2}=0$.

(iii) there exist matrices $Y_{1}, Y_{2} \in \mathbb{S}^{2 n}$ and $X_{1}, X_{2} \in$ $\mathbb{R}^{2 n \times 2 n}$ such that (6) holds.

(iv) there exist matrices $Y_{1}, Y_{2} \in \mathbb{S}^{2 n}, X_{1}, X_{2} \in \mathbb{R}^{2 n \times 2 n}$ and $Z \in \mathbb{R}^{2 n \times n}$ such that (8) holds with $\bar{\Gamma}=g_{1}$, where $g_{1}$ is given in (16).

(v) there exist matrices $Y_{1}, Y_{2} \in \mathbb{S}^{2 n}, X_{1}, X_{2} \in \mathbb{R}^{2 n \times 2 n}$ and $Z \in \mathbb{R}^{2 n \times 2 n}$ such that (8) holds with $\bar{\Gamma}=\left[\begin{array}{l}g_{1} \\ g_{2}\end{array}\right]$, where $g_{1}$ and $g_{2}$ are given in (16).

(vi) there exist matrices $N_{1}, N_{2}$ in $\mathbb{R}^{7 n \times n}$ such that (3) holds.

Proof : In order to assess stability of system (14), we consider the Lyapunov-Krasovskii functional $V\left(x_{t}, \dot{x}_{t}\right)$ introduced in [13]:

$$
V\left(x_{t}, \dot{x}_{t}\right)=V_{1}\left(x_{t}\right)+V_{2}\left(x_{t}\right)+V_{3}\left(x_{t}, \dot{x}_{t}\right),
$$

where

$$
\begin{aligned}
V_{1}\left(x_{t}\right)= & {\left[\begin{array}{c}
x(t) \\
\int_{t-h_{1}}^{t} x(s) \mathrm{d} s \\
\int_{t-h_{2}}^{t-h_{1}} x(s) \mathrm{d} s
\end{array}\right]^{T} P\left[\begin{array}{c}
x(t) \\
\int_{t-h_{1}}^{t} x(s) \mathrm{d} s \\
\int_{t-h_{2}}^{t-h_{1}} x(s) \mathrm{d} s
\end{array}\right], } \\
V_{2}\left(x_{t}\right)= & \int_{t-h_{1}}^{t} x^{T}(s) S_{1} x(s) \mathrm{d} s \\
& +\int_{t-h_{1}}^{t-h_{1}} x^{T}(s) S_{2} x(s) \mathrm{d} s \\
V_{3}\left(x_{t}, \dot{x}_{t}\right)= & h_{1} \int_{-h_{1}}^{0} \int_{t+\theta}^{t} \dot{x}^{T}(s) R_{1} \dot{x}(s) \mathrm{d} s \\
& +h_{12} \int_{-h_{2}}^{-h_{1}} \int_{t+\theta}^{t} \dot{x}^{T}(s) R_{2} \dot{x}(s) \mathrm{d} s
\end{aligned}
$$

Following the same procedure as used in [13] and applying Wirtinger-based inequality [10], we obtain the 


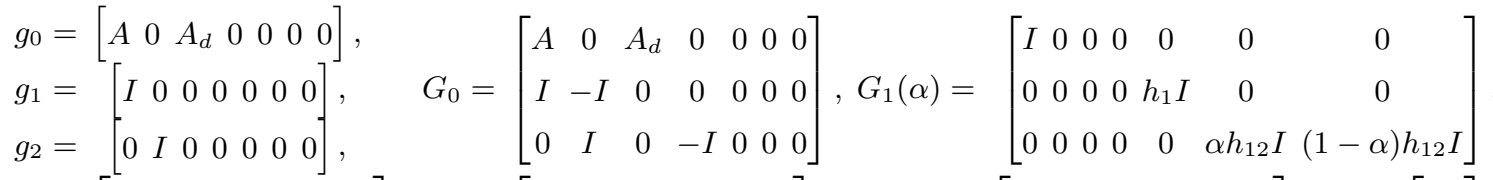

$$
\begin{aligned}
& G_{2}=\left[\begin{array}{ccccccc}
I & -I & 0 & 0 & 0 & 0 & 0 \\
I & I & 0 & 0 & -2 I & 0 & 0
\end{array}\right], G_{3}=\left[\begin{array}{ccccccc}
0 & I & -I & 0 & 0 & 0 & 0 \\
0 & I & I & 0 & 0 & -2 I & 0
\end{array}\right], \quad G_{4}=\left[\begin{array}{ccccccc}
0 & 0 & I & -I & 0 & 0 & 0 \\
0 & 0 & I & I & 0 & 0 & -2 I
\end{array}\right] . \quad \Gamma=\left[\begin{array}{l}
G_{3} \\
G_{4}
\end{array}\right] \text {. }
\end{aligned}
$$

\begin{tabular}{|l|r|c|c|c||c|}
\hline$h_{1}$ & 0.0 & 0.4 & 0.7 & 1.0 & No. of var. \\
\hline$[8]$ & 1.86 & 1.88 & 1.95 & 2.06 & $3.5 n^{2}+2.5 n$ \\
{$[2]$} & 1.86 & 1.89 & 1.98 & 2.12 & $11.5 n^{2}+3.5 n$ \\
{$[13]$} & 2.11 & 2.17 & 2.23 & 2.31 & $10.5 n^{2}+3.5 n$ \\
{$[9]$} & 2.14 & 2.19 & 2.24 & 2.31 & $21 n^{2}+6 n$ \\
{$[16]$} & 2.18 & 2.21 & 2.25 & 2.32 & $54.5 n^{2}+9.5 n$ \\
\hline \hline Th.1 & & & & & \\
\hline (i) [19] & 2.20 & 2.25 & 2.286 & 2.345 & $10.5 n^{2}+3.5 n$ \\
(ii) [18, 20] & 2.21 & 2.25 & 2.286 & 2.345 & $14.5 n^{2}+3.5 n$ \\
(iii) & 2.23 & 2.264 & 2.289 & 2.345 & $18.5 n^{2}+5.5 n$ \\
(iv) & 2.243 & 2.269 & 2.291 & 2.345 & $20.5 n^{2}+5.5 n$ \\
(v) & 2.243 & 2.272 & 2.294 & 2.347 & $22.5 n^{2}+5.5 n$ \\
(vi) & 2.243 & 2.272 & 2.294 & 2.347 & $30 n^{2}+1.5 n$ \\
\hline
\end{tabular}

\section{Table 1}

Example 1: Admissible upper bound of $h_{2}$ for different $h_{1}$

derivative of the functionals along the trajectories of the system

$$
\dot{V}\left(x_{t}, \dot{x}_{t}\right) \leq \zeta^{\top}(t)\left(\Phi_{0}(\alpha)-\Theta(\alpha)\right) \zeta(t)
$$

where $\Phi_{0}(\alpha)$ is given by (17), $\Theta(\alpha)$ is defined in (1) with $R=\mathcal{R}_{2}, \alpha=\frac{h-h_{1}}{h_{12}}$ and $\Gamma$ given in (16) and where $\zeta(t)=\left[\zeta_{1}^{\top}(t), \zeta_{2}^{\top}(t)\right]^{\top}$ with

$$
\zeta_{1}(t)=\left[\begin{array}{c}
x(t) \\
x\left(t-h_{1}\right) \\
x(t-h) \\
x\left(t-h_{2}\right)
\end{array}\right], \zeta_{2}(t)=\left[\begin{array}{c}
\frac{1}{h_{1}} \int_{t-h_{1}}^{t} x^{\top}(s) \mathrm{d} s \\
\frac{1}{h-h_{1}} \int_{t-h}^{t-h_{1}} x^{\top}(s) \mathrm{d} s \\
\frac{1}{h_{2}-h} \int_{t-h_{2}}^{t-h} x^{\top}(s) \mathrm{d} s
\end{array}\right]
$$

We are now in a position to apply Corollary 1 or Lemma 2 to conclude the proof.

Remark 5 It is worth noting that Theorem 1 includes the recent conditions provided in [19] and [18, 20] in the particular cases (i) and (ii), respectively, which demonstrates again the generality of the formulation proposed in this paper.

\section{$5 \quad$ Illustrative examples}

Example 1: Consider the following much-studied linear time-delay systems (14) with

$$
A=\left[\begin{array}{cc}
-2 & 0 \\
0 & -0.9
\end{array}\right], A_{d}=\left[\begin{array}{cc}
-1 & 0 \\
-1 & -1
\end{array}\right]
$$

Table 1 shows the maximum admissible upper bound of $h_{2}$ for different values of $h_{1}$ obtained by Theorem 1 and several methods from the literature. From Table 1 it is seen that our results provided in the present paper are favorably compared with [13]. Indeed the only difference between these two works is that the reciprocally convex quadratic term $\Theta$ given by (1) is bounded in Theorem 1 by Lemma 2 or Corollary 1 and in [13] by the original reciprocally convex combination lemma [8].

It is also worth noting that conditions (iv)-(vi) of Theorem 1 leads to the same results even if the computational complexities of the stability conditions are different. It is verified again that the use of additional slack matrices in condition (vi) are not necessary to reduce the conservatism. Moreover, it is possible to reduce the conservatism of the stability conditions by considering more accurate integral inequalities (see e.g., $[4,6,9,11,20]$ ), but this will not be presented since it is out of the scope of this paper.

Example 2: Consider system (14) borrowed from [9] with the following matrices

$$
A=\left[\begin{array}{cc}
0 & 1 \\
-10 & -1
\end{array}\right], \quad A_{1}=\left[\begin{array}{cc}
0 & 0.1 \\
0.1 & 0.2
\end{array}\right]
$$

Table 2 presents admissible upper bound of $h_{2}$ for different $h_{1}$. We can see that the method of Theorem 1 is competitive with the stability conditions from $[9,13,16]$, which demonstrates, again, the potential of Lemma 2 or Corollary 1. Moreover, it is observed that for values of $h_{1}=\{0.7,1.0\}$, the best results are obtained by [9]. This improvement of [9] can be explained by the use of the auxiliary function integral inequality, which is less conservative than Wirtinger-based inequality. 


\begin{tabular}{|l|c|c|c|c|c|}
\hline$h_{1}$ & 0.0 & 0.3 & 0.7 & 1.0 & 2.0 \\
\hline \hline$[13]$ & 1.59 & 2.01 & 2.41 & 2.62 & 3.59 \\
{$[9]$} & 1.64 & 2.13 & 2.70 & 2.96 & 3.63 \\
{$[16]$} & 1.80 & 2.19 & 2.58 & 2.79 & 3.68 \\
\hline \hline Th.1 (i) & 1.72 & 2.14 & 2.55 & 2.75 & 3.71 \\
Th.1 (ii) & 1.76 & 2.18 & 2.59 & 2.79 & 3.75 \\
Th.1 (iii) & 1.80 & 2.22 & 2.63 & 2.83 & 3.793 \\
Th.1 (iv)-(vi) & 1.862 & 2.288 & 2.695 & 2.895 & 3.849 \\
\hline
\end{tabular}

Table 2

Example 2: Admissible upper bound of $h_{2}$ for different $h_{1}$

\section{Conclusions}

By reformulating an equivalent form of Moon et al.'s inequality, this paper provided more insights on the relationship between some existing matrix inequalities. It was demonstrated that some existing inequalities can be captured as particular cases of Moon et al.'s inequality. Examples show the best tradeoff between the reduction of conservatism and the numerical complexity. Notice that the objective of this paper was not to provide the least conservative stability conditions, but mainly to establish the existing links between several matrix inequalities provided in the literature. However, the proposed analysis can be largely improved by considering other integral inequalities or other functionals as proposed for instance in $[4,11,20]$.

\section{References}

[1] E. Fridman. Introduction to time-delay systems: Analysis and control. Springer, 2014.

[2] E. Fridman, U. Shaked, and K. Liu. New conditions for delay-derivative-dependent stability. Automatica, 45(11):2723-2727, 2009.

[3] L. V. Hien and H. Trinh. Refined Jensen-based inequality approach to stability analysis of timedelay systems. IET Control Theory $\&$ Applications, 9(14):2188-2194, 2015.

[4] S. Y. Lee, W. I. Lee, and P. G. Park. Polynomialsbased integral inequality for stability analysis of linear systems with time-varying delays. Journal of the Franklin Institute, 354(4):2053-2067, 2017.

[5] K. Liu and A. Seuret. Comparison of bounding methods for stability analysis of systems with timevarying delays. Journal of the Franklin Institute, 354(7):2979-2993, 2017.

[6] K. Liu, A. Seuret, and Y. Q. Xia. Stability analysis of systems with time-varying delays via the second-order Bessel-Legendre inequality. Automatica, 76:138-142, 2017.

[7] Y. S. Moon, P. G. Park, W. H. Kwon, and Y. S. Lee. Delay-dependent robust stabilization of uncertain state-delayed systems. International Journal of Control, 74(14):1447-1455, 2001.
[8] P. Park, J. Ko, and C. Jeong. Reciprocally convex approach to stability of systems with time-varying delays. Automatica, 47(1):235-238, 2011.

[9] P. G. Park, W. I. Lee, and S. Y. Lee. Auxiliary function-based integral inequalities for quadratic functions and their applications to time-delay systems. Journal of the Franklin Institute, 352(4):1378-1396, 2015.

[10] A. Seuret and F. Gouaisbaut. Wirtinger-based integral inequality: Application to time-delay systems. Automatica, 49(9):2860 - 2866, 2013.

[11] A. Seuret and F. Gouaisbaut. Hierarchy of LMI conditions for the stability of time delay systems. Systems \& Control Letters, 81:1-7, 2015.

[12] A. Seuret and F. Gouaisbaut. Allowable delay sets for the stability analysis of linear time-varying delay systems using a delay-dependent reciprocally convex lemma. In Proceedings of the 20th IFAC World Congress, Toulouse, France, July 2017.

[13] A. Seuret, F. Gouaisbaut, and E. Fridman. Stability of systems with fast-varying delay using improved Wirtinger's inequality. In Proceedings of the 52nd IEEE CDC, pages 946-951, Florence, Italy, December 2013.

[14] H. Shao. New delay-dependent stability criteria for systems with interval delay. Automatica, 45(3):744749, 2009.

[15] S. Xu and J. Lam. A survey of linear matrix inequality techniques in stability analysis of delay systems. International Journal of Systems Science, 39(12):1095-1113, 2008.

[16] H. B. Zeng, Y. He, M. Wu, and J. H. She. Freematrix-based integral inequality for stability analysis of systems with time-varying delay. IEEE Transactions on Automatic Control, 60(10):2768-2772, 2015.

[17] C. K. Zhang, Y. He, L. Jiang, and M. Wu. Notes on stability of time-delay systems: bounding inequalities and augmented Lyapunov-Krasovskii functionals. IEEE Transactions on Automatic Control, 62(10):5331-5336, 2017.

[18] C. K. Zhang, Y. He, L. Jiang, M. Wu, and Q. G. Wang. An extended reciprocally convex matrix inequality for stability analysis of systems with timevarying delay. Automatica, 85:481-485, 2017.

[19] C. K. Zhang, Y. He, L. Jiang, M. Wu, and H. B. Zeng. Stability analysis of systems with timevarying delay via relaxed integral inequalities. Systems \&6 Control Letters, 92:52-61, 2016.

[20] X. M. Zhang, Q. L. Han, A. Seuret, and F. Gouaisbaut. An improved reciprocally convex inequality and an augmented Lyapunov-Krasovskii functional for stability of linear systems with time-varying delay. Automatica, 84:221-226, 2017. 